\title{
Values of Large Market Games ${ }^{1}$ )
}

\author{
By S. Hart, Stanford ${ }^{2}$ )
}

\begin{abstract}
Three aspects of the application of the game theoretic concept of "value" to non-atomic economies - such as markets or production - are studied: first, the relation between value and equilibria; second, the problems of existence and non-existence of value; and third, a new way of defining value for these games, in order to guarantee its existence, which leads to interesting economic interpretations.
\end{abstract}

\section{Introduction}

The relations between game theoretic and economic concepts have been studied for a long time, trying to get a better insight into the laws governing the behavior of economic agents.

Much interest has been devoted to "large" economies ${ }^{3}$ ), where the individual is "negligible". Such situations are called "perfectly competitive", and the appropriate economic concept is that of "competitive equilibrium".

The first game theoretic solution studied in this context is the core, the main result being:

Core Equivalence Theorem: In a perfectly competitive economy, the core and the set of competitive allocations coincide [cf. Debreu/Scarf; Aumann [1964]; Vind; Hildenbrand, and others].

The next most used concept is the (Shapley) value - in particular, since it captures traditional economic ideas of "marginal contribution" (or, "worth"). The corresponding result is the following:

Value Theorem: In a perfectly competitive economy, every value allocation is competitive, and the two sets of allocations coincide if the economy is "sufficiently differentiable".

There are two main ways to model perfect competition. One is a limit approach, where sequences of finite economies, increasing in size, are considered (e.g., replicas). The other is using a non-atomic continuum as the space of agents.

\footnotetext{
1) This work was supported by National Science Foundation Grant SOC75-21820 at the Institute for Mathematical Studies in the Social Sciences, Stanford University. Presented at the International Conference on Applied Game Theory, Vienna, June 1978.

${ }^{2}$ ) Sergiu Hart; Stanford University, Institute for Mathematical Studies in the Social Sciences. Stanford, California 94305, USA.

${ }^{3}$ ) By "economy" we mean a market, or a production economy - as in Section 2.
} 
Also, the two kinds of economic models are studied: Walrasian exchange (markets without transferable utility), and "monetary" markets (with transferable utility). As it will be pointed out in Section 2, the latter also represents production economies.

The following table summarizes the research done on the Value Theorem:

\begin{tabular}{l|l|l|l} 
& $\begin{array}{l}\text { Limit of Finite } \\
\text { Economies }\end{array}$ & $\begin{array}{l}\text { Non-atomic } \\
\text { Economies }\end{array}$ & \\
\hline $\begin{array}{l}\text { Monetary (with } \\
\text { transferable } \\
\text { utility) }\end{array}$ & Shapley [1964] & Aumann/Shapley [1974] & Differentiable \\
\cline { 2 - 4 } & Champsaur [1975] & Hart $[1977 \mathrm{~b}]$ & Non-differentiable \\
\hline $\begin{array}{l}\text { Walrasian (with- } \\
\text { out transferable } \\
\text { utility) }\end{array}$ & Mas-Colell $[1977]$ & Aumann $[1975]$ & Differentiable \\
\cline { 2 - 4 } & Champsaur $[1975]$. & Hart $[1977 \mathrm{~b}]$ & Non-differentiable \\
\hline
\end{tabular}

Tab. 1

In this paper we deal with non-atomic economies. After presenting the basic models and defining the generalized asymptotic values in Section 2, we divide the results in three parts. The first one (Section 3) is devoted to the Value Theorem; the second one (Section 4), to the existence (and non-existence) of the asymptotic value; and in the last one (Section 5), we try to overcome these problems by defining a new measurebased value:

\section{Preliminaries}

This section includes the basic models of non-atomic economies, the definitions of (generalized) asymptotic values, and some preliminary results.

We start by describing a non-atomic economy (market) - as in Aumann/Shapley [1974], Aumann [1975], and Hart [1977b].

The trader space is a measurable space $(I, C)$, which we assume to be standard $\left.{ }^{4}\right)$. A non-negative, $\sigma$-additive and non-atomic measure $\mu$ on $C$ is given, called the population measure. To simplify our notations, we will sometimes write $\left.{ }^{5}\right) \int_{S} f d \mu$ and $\int_{S} f$ to mean
$\int f(t) d \mu(t)$. $\int_{S} f(t) d \mu(t)$.

The commodity space is $\Omega$, the non-negative orthant of the $l$-dimensional Euclidean space $\mathbf{R}^{l}$, where $l$ is the number of commodities. For $x$ in $\mathbf{R}^{l}, x^{j}$ will denote its $j$-th coordinate.

The initial allocation $\underset{\sim}{a}$ is an integrable function from $I$ to $\Omega$. We assume that every commodity is actually present in the market, i.e.,

\footnotetext{
$\left.{ }^{4}\right)$ I.e., isomorphic to the unit interval with the Borel $\sigma$-field. This assumption is not too restrictive, since any uncountable Borel subset of any Euclidean space, and indeed of any complete separable metric space, with the corresponding Borel $\sigma$-field, is standard - cf. Proposition (1.1) in Aumann/Shapley [1974].

${ }^{5}$ ) Letters with 'wiggle' underneath will denote function defined on $I$.
} 


$$
\int_{I^{\sim}} a^{j}>0, \text { for all } 1 \leqslant j \leqslant l
$$

(commodities with no initial supply can be obviously ingnored).

An allocation is an integrable function $\underset{\sim}{x}$ from $I$ to $\Omega$; such that $\underset{I}{x} \underset{I}{x}=\int_{I} a$.

Here we distinguish between the two kinds of economies: monetary and Walrasian.

In the transferable utility case (monetary markets), to each $t$ in $I$ there corresponds a real-valued function $u_{t}$ defined on $\Omega$, called the utility function of $t$. All these functions are normalized by $u_{t}(0)=0$, and they further satisfy:

(2.2) $x \geqslant y$ implies $\left.^{6}\right) u_{t}(x) \geqslant u_{t}(y)$ (weak monotonicity),

(2.3) $u_{t}$ is a continuous function (continuity),

(2.4) $u_{t}(x)$, as a function of the pair $(t, x)$, is measurable in the product field $C \times B^{l}$, where $B^{l}$ denotes the Borel $\sigma$-field on $\Omega$ (measurability), and

(2.5) $u_{t}(x)=o(\|x\|)$ as $\|x\| \rightarrow \infty$, integrably in $t$ (i.e., for every $\epsilon>0$ there is an integrable function $\eta$ defined on $I$, such that $\left|u_{t}(x)\right| \leqslant \epsilon\|x\|$ whenever $\|x\| \geqslant \underset{\sim}{\eta}(t))$.

Given the above economy, we will define the corresponding market game $\mathrm{v}$ by

$$
\mathrm{v}(S)=\max \left\{\int_{S} u_{t}(\underset{\sim}{x}(t)) d \mu(t) \mid \int_{S} \underset{\sim}{x}=\int_{S} \underset{\sim}{a} \text { and } \underset{\sim}{x}(t) \in \Omega \text { for all } t \in S\right\},
$$

for all $S \in \mathcal{C}$, the maximum being attained by the main theorem in Aumann/Perles [1965]. Then $\mathrm{v}$ is a non-atomic game with side payments, and further it belongs to the space $H_{+}^{\prime}$, as defined in Hart [1977a, Section 2]; namely, it is the limit in the supremum norm of polynomials of non-atomic measures, it is superadditive, monotone, and homogeneous of degree one [see Proposition (3.4) in Hart, 1977b].

In terms of our exchange market, the interpretation of $\mathrm{v}(S)$ is as follows: there is an additional commodity, called "money", such that each trader's utility increases by one unit for each one unit of money. The maximum utility the coalition $S$ can get, by reallocating its own initial resources between its members, is then exactly v $(S)$.

The second interpretation of this model is that of a production economy. There are $l$ "inputs" (i.e., raw goods), and one "output" (i.e., a finished good). Each participant $t$ can produce out of a vector $x$ (in $\Omega$ ) of inputs, an amount $u_{t}(x)$ of the output $\operatorname{good}^{7}$ ). The initial allocation of raw goods being $\underset{\sim}{a}, \mathrm{v}(S)$ is then the maximal quantity of the finished good that $S$ can produce, again by using its own resources alone.

A transferable utility competitive equilibrium (t.u.c.e.) is a pair $(\underset{\sim}{x}, p)$, where $\underset{\sim}{x}$ is an allocation and $p$ is a price vector in $\Omega$, such that

$$
u_{t}(x)-p \cdot(x-\underset{\sim}{a}(t)) \leqslant u_{t}(\underset{x}{x}(t))-p \cdot(\underset{\sim}{x}(t)-\underline{a}(t))
$$

\footnotetext{
${ }^{6}$ ) For $x$ and $y$ in $\Omega, x \geqslant y$ means $x^{j} \geqslant y^{j}$ for all $1 \leqslant j \leqslant l$.

7) A more precise interpretation will be that a producer $d t$ gets $u_{t}(x) \mu(d t)$ out of $x \mu(d t)-\mathrm{cf}$. Section 30 in Aumann/Shapley [1974].
} 
for all $x$ in $\Omega$ and (almost) all $t$ in $I$. The corresponding competitive payoff distribution is the measure $\left.{ }^{8}\right) \nu_{p}$ defined by

$$
\nu_{p}(S)=\int_{S}\left[u_{t}(\underset{\sim}{x}(t))-p \cdot(\underset{\sim}{x}(t)-\underset{\sim}{a}(t))\right] d \mu(t),
$$

for all $S \in \mathrm{C}$.

The t.u.c.e. is actually a usual (Walrasian) competitive equilibrium, the price of "money" being normalized to 1 - see Section 32 in Aumann/Shapley [1974] for a more detailed discussion.

From now on, $P$ will always denote the set of all equilibrium prices, i.e., the set of all $p$ in $\Omega$ such that $(\underset{\sim}{x}, p)$ is a t.u.c.e. for some allocation $\underset{\sim}{x}$.

Proposition 2.9:

(i) $\quad P$ is a non-empty, convex and compact subset of $\Omega$.

(ii) $\underset{\sim}{x}$ is a t.u.c.e. allocation if and only if $\mathrm{v}(I)$ is attained $\left.{ }^{9}\right)$ at $\underset{\sim}{x}$. Moreover, $(\underset{\sim}{x}, p)$ is then a t.u.c.e. for all $p$ in $P$, and the corresponding competitive payoff distribution does not depend on $\underset{x}{x}$ (i.e., is the same for all such $\underline{x}^{10}$ ).

(iii) The core of $v$ and the set of competitive payoff distributions coincide.

Proof: Propositions 32.2 and 32.5 in Aumann/Shapley [1974], Propositions (2.10) and (2.20) in Hart [1977b], and Theorem 23.4 in Rockafellar [1970].

The second model is that of a Walrasian non-atomic market. Unlike the previous case, no (cardinal) utility functions are given. Instead, for each $t$ in $I$ there is an (ordinal) preference relation $\gg_{t}$ on $\Omega$, satisfying:

(2.10) $x \geqslant y$ and $x \neq y$ imply $x \gg_{t} y$ (desirability),

(2.11) for each $x$ in $\Omega$, the sets $\left\{y \mid y \gg_{t} x\right\}$ and $\left\{y \mid x \gg_{t} y\right\}$ are open relative to $\Omega$ (continuity), and

(2.12) for any two measurable functions $x$ and $y$ from $I$ to $\Omega$, the set $\left\{t \mid \underset{\sim}{x}(t) \gg_{t} \underset{\sim}{y}(t)\right\}$ belongs to $\mathrm{C}$ (measurability).

A competitive equilibrium is a pair $(\underset{x}{x} p)$, where $x$ is an allocation and $p \neq 0$ is a price vector in $\Omega$, such that $\underset{\sim}{x}(t)$ is maximal with respect to $\gg_{t}$ in the budget set of trader $t$

$$
B_{p}(t)=\{x \in \Omega \mid p \cdot x \leqslant p \cdot \underset{a}{a}(t)\},
$$

for (almost) all $t$ in $I$.

We come now to the definition of value - first in the transferable utility case - using the asymptotic approach, due to Kannai [1966].

${ }^{8}$ ) Because of Proposition 2.9, we can use the notation $\nu_{p}$ (instead of $\nu_{(\underset{\sim}{x}, p)}$ ).

$\left.{ }^{9}\right)$ I.e., $\mathrm{v}(I)=\int_{I} u_{t}(\underset{\sim}{x}(t))$ and $\int_{I} \underset{\sim}{x}=\int_{I}^{a}$.

${ }^{10}$ ) But it does depend on $p$. 
Let $\mathrm{v}$ be a non-atomic game on the measurable space $(I, C)$ (i.e., $\mathrm{v}$ is a real function on $C$ with $\vee(\emptyset)=0$ ).

A partition $\Pi$ of $(I, C)$ is a finite family of subsets of $I$, which are measurable (i.e., belong to $C$ ) and disjoint, and whose union is $I$. A sequence $P=\left\{\Pi_{m}\right\}_{m=1}^{\infty}$ of partitions is called admissible if it satisfies

(2.13) it is decreasing, i.e. for all $m$, each member of $\Pi_{m}$ is a union of members of $\Pi_{m+1} ;$ and

(2.14) it is separating, i.e. for each $s, t$ in $I, s \neq t$, there is $m$ such that $s$ and $t$ are in different members of $\Pi_{m}$.

For a given partition $\Pi$, let $v_{\Pi}$ denote the finite game derived from $v$, whose players are the members of $\Pi$, namely

$$
\mathrm{v}_{\Pi}(\Lambda) \equiv \mathrm{v}(\underset{B \in \Lambda}{\cup} B)
$$

for all $\Lambda \subset \Pi$.

Let $T \in C$ and let $P=\left\{\Pi_{m}\right\}$ be an admissible sequence of partitions whose first term is $\Pi_{1}=\{T, I \backslash T\}$. For each $m$, let $T_{m}=T_{\Pi_{m}}$ be the coalition corresponding to $T$ in $\mathrm{v}_{\Pi_{m}}$, namely $T_{m}=\left\{B \in \Pi_{m} \mid B \subset T\right\}$. Let $\phi \mathrm{v}_{\Pi_{m}}$ denote the Shapley value of the finite game $v_{\Pi_{m}}$. If the numbers $\left(\phi v_{\Pi_{m}}\right)\left(T_{m}\right)$ approach a limit as $m \rightarrow \infty$, and this limit is independent of the sequence $P$, then it will be denoted by $(\phi v)(T)$. It $(\phi v)(T)$ exists for all $T \in C$, then the function $\phi \mathrm{v}$ will be called the asymptotic value of $\mathrm{v}$.

In view of the non-existence of the asymptotic value in some cases of interest (see Section 4), we will also consider generalized asymptotic values, where the limit of $\left(\phi \mathrm{v}_{T_{m}}\right)\left(T_{m}\right)$ should exist and be the same for all $P$ in a given class of admissible sequences. Examples of such values are the measure based values, to be defined in Section 5. The reason for this definition is that the Value Theorem holds for any generalized asymptotic value (and not only for the usual one), as shown in Section 3. A last immediate remark is that the asymptotic value exists if and only if all generalized asymptotic values are identical.

In what regards the non-transferable utility case, we will use the following NashHarsanyi-Shapley procedure [cf. Harsanyi; Shapley 1969; Aumann, 1975].

Let $U=\left\{u_{t}\right\}_{t \in I}$ be a family of utility functions, representing the given preferences $\left\{\gg_{t}\right\}_{t \in I}$, namely,

$$
u_{t}(x)>u_{t}(y) \text { if and only if } x \gg_{t} y,
$$

for all $t$ in $I, x$ and $y$ in $\Omega$. If $U$ also satisfies (2.5), a transferable utility market $\mathrm{v} \equiv \mathrm{v}_{U}$ can be defined by (2.6) (note that (2.2), (2.3) and (2.4) are implied by (2.10), (2.11) and (2.12), respectively). 
An allocation $\underset{\sim}{x}$ is called a (generalized) asymptotic value allocation if there exists a family $U$ of utilities, satisfying (2.5) and (2.15), such that $\mathrm{v}_{U}$ has a (generalized) asymptotic value $\phi \mathrm{v}_{U}$, and

$$
\left(\phi \mathrm{v}_{U}\right)(S)=\int_{S} u_{t}(\underset{\sim}{x}(t)) d \mu(t),
$$

for all $S$ in $C$.

For discussions of this approach, the reader is referred to the above noted papers of Shapley [1969] and Aumann [1975].

\section{The Value Theorem}

The results in this section are, the same as those in Hart [1977b], extended here to the generalized asymptotic values (using essentially the same proofs).

We start with the monetary markets.

Theorem 3.1: Let $\mathrm{v}$ be a market game arising from a non-atomic economy with transferable utility, satisfying (2.1) - (2.5). Let $\phi \mathrm{v}$ be a generalized asymptotic value. Then $\phi \mathrm{v}$ is a competitive payoff distribution.

Proof: By Proposition (3.4) in Hart [1977b], v belongs to $H_{+}^{\prime}$. Applying now Proposition (7.1) and (5.4) in Hart [1977a], we get

$$
(\phi v)(T)=\lim _{m \rightarrow \infty}\left(\phi v_{\Pi_{m}}\right)\left(T_{m}\right) \geqslant \partial \mathrm{v}^{*}\left(\chi_{I} ; \chi_{T}\right) \geqslant \mathrm{v}(T),
$$

from which it follows that $\phi \mathrm{v}$ belongs to the core of $\mathrm{v}$, hence by Proposition 2.9 (iii) it is a competitive payoff distribution.

The second part of the Value Theorem assumes differentiability. The following theorem is actually stronger than the asymptotic part of the results of Aumann/Shapley [1974] (see Table 1).

Theorem 3.2: Let $v$ be a market game arising from a non-atomic economy with transferable utility, satisfying $(2.2)-(2.5)$ and

(3.3) for every $t$ in $I$ and $1 \leqslant j \leqslant l$, $\partial u_{t}(x) / \partial x^{j}$ exists at every $x$ in $\Omega$ with $x^{j}>0$.

Then the set of competitive payoff distributions and the set of (generalized) asymptotic values of $v$ concide, and consist of one element only.

Proof: Theorem D in Hart [1977b].

We come now to the non-transferable utility case.

Theorem 3.4: In a non-atomic Walrasian market satisfying (2.1) and (2.10) - (2.12), every generalized asymptotic value allocation is competitive. 
Proof: The same proof as that of Theorem E in Hart [1977b], using Theorem 3.1 above instead of Theorem A there.

\section{Existence of Asymptotic Value}

Since the value for the Walrasian markets, by its definition, depends on the existence of value in the transferable utility case, we will deal in the next two sections with the latter only.

We start with some "positive" results.

Theorem 4.1: Let $\mathrm{v}$ be a market game arising from a non-atomic economy with transferable utility, satisfying $(2.1)-(2.5)$. If there is a unique competitive payoff distribution, then $v$ has an asymptotic value.

Proof: Theorem C in Hart [1977a], Proposition (3.4) in Hart [1977b], and Proposition 2.9 (iii).

The next theorem is a "generic existence theorem".

Theorem 4.2: Given utility functions $U=\left\{u_{t}\right\}_{t \in I}$ satisfying (2.2) $-(2.5)$, let $A \equiv A_{U}$ be the set of all vectors $a$ in $\Omega$ such that there is a transferable utility non-atomic market $(\underset{a}{a}, U)$ with $\int_{I} \underset{a}{a}=a$, for which the asymptotic value does not exist. Then $A$ is a set of Lebesgue measure zero in $\Omega$.

Proof: See the proof of Theorem C in Hart [1977b].

Remark 4.3: Actually, a stronger result is proved as Theorem C in Hart [1977b], namely, that the set of competitive payoff distributions coincides with the asymptotic value "almost everywhere" (which is defined in the same way as in the above Theorem 4.2).

However, in general, the asymptotic value need not exist. A necessary condition is given in the next theorem. For a subset $X$ of a linear space, $x_{0}$ is a center of symmetry of $X$ if, for every $x$ in $X$, its symmetric image with respect to $x_{0}, 2 x_{0}-x$, belongs also to $X$.

To avoid inessential complications, we will assume that the excess demand in equilibrium has full dimension, namely, that given a t.u.c.e. allocation $\underset{\sim}{x}$, the linear (affine) subspace $L(\underset{\sim}{x}-\underset{\sim}{a})$ of $\mathbf{R}^{l}$ spanned by all vectors $\int_{S}(\underset{\sim}{x}-\underset{\sim}{a}) d \mu$, for $\underset{\sim}{\sim} S \in C$, has full dimension (i.e., dimension $l$ ). In case this is not satisfied, $P$ (the set of equilibrium prices) should be replaced by its projection on $L(\underset{\sim}{x}-\underset{\sim}{a})$.

Theorem 4.4: Let $\mathrm{v}$ be a market game arising from a non-atomic economy with transferable utility satisfying $(2.1)-(2.5)$. If $v$ has an asymptotic value, then the set of competitive payoff distributions and the set $P$ of equilibrium prices each have a center of symmetry. 
Proof: Theorem B in Hart [1977b] (see also Added in Proof (2)' there).

As an example where this condition is not satisfied (hence, there is no asymptotic value) - one can consider the "three-handed glove market" [cf. Aumann/Shapley, p. 203]. It should be also noted that the above condition is necessary but not sufficient [cf. example 8.1 in Hart, 1977a].

\section{Measure-Based Values}

In order to get a value for all market games, we will define-in this section a specific generalized asymptotic value [see Hart, 1978].

First, let us consider the reasons for the non-existence of the asymptotic value. The definition requires that for all admissible sequences, the limit of the Shapley values of the corresponding finite games should exist, and be independent of the particular sequence chosen. In all the cases studied in this context, admissible sequences with dif ferent limits have been constructed. A deeper look reveals that all those partitions consisted of one (small) set which was "much bigger" than all the others. For example, consider the partition of $[0,1]$ into one interval of length $1 / n$ and $n(n-1)$ intervals of length $1 / n^{2}$, and let $n \rightarrow \infty$.(to ensure that the partitions get "finer", one can take $n=2^{m}$ and $\left.m \rightarrow \infty\right)$.

In case the only "data" is a game $v$, there is nothing that can make the above sequence of partitions "inadmissible". However, when one is considering an economy, and $\mathrm{v}$ is the derived "market game", additional data is given - namely, an underlying "population measure" $\mu$. E.g., assume $[0,1]$ to be the set of traders, and $\mu$ the Lebesque measure. Then the sequence of partitions described above does not seem to be a good approximation of the traders' space (some of them being always given much more weight than others!).

This indicates the way to proceed in order to guarantee the existence of the value. It was first used in Aumann/Kurz [1977], by restricting the admissible partitions to have all their elements equal in $\mu$-measure. Here we adopt a slightly more "liberal" approach, requiring the measure of the elements of the partitions to get "close" one to another as $m \rightarrow \infty$.

Formally, given a measure $\mu$ on $(I, C)$, a sequence $P=\left\{\Pi_{m}\right\}_{m=1}^{\infty}$ of partitions is called $\mu$-admissible if it is admissible (i.e., satisfies (2.13) and (2.14)), and furthermore

$$
\lim _{m \rightarrow \infty} \frac{\max _{B \in \Pi_{m}} \mu(B)}{\min _{B \in \Pi_{m}} \mu(B)}=1
$$

The generalized asymptotic value corresponding to the class of all $\mu$-admissible sequences is called $\mu$-based value, or $\mu$-value.

In order to guarantee existence of the $\mu$-value for a market game, we need one further assumption, which can be interpreted as an added "competitiveness" requirement: that the variance of the excess demand, in equilibrium, should be finite. In- 
tuitively, this implies that no arbitrarily "small" group of traders can have an arbitrarily "large" excess demand (recall that the total - hence, average - excess demand in equilibrium is zero). Usually, all allocations will be bounded, therefore this assumption will be surely satisfied.

We can now state our main result. As in Section 4, we will make the simplifying assumption that $L(\underset{\sim}{x}-\underset{\sim}{a})$ has full dimension (see Theorem 4.4; in the degenerate case, replace $P$ by proj $L\left(\underset{\sim}{x-a} \underset{\sim}{a} P\right.$ and $\mathrm{R}^{l}$ by $\left.L(\underset{\sim}{x}-\underline{a})\right)$.

Theorem 5.2: Let $\mathrm{v}$ be a market game arising from a non-atomic economy with transferable utility, satisfying $(2.1)-(2.5)$. Let $P$ be the set of all equilibrium prices, and

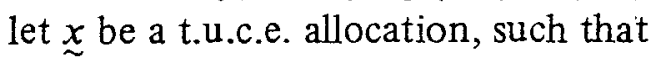

$$
\int_{I}\left(z_{\sim}^{j}\right)^{2} d \mu<\infty, \text { for all } 1 \leqslant j \leqslant l
$$

and $L(z)$ has full dimension, where $\underset{\sim}{z} \equiv \underset{\sim}{x}-\underset{\sim}{a}$.

Then v has a $\mu$-value $\phi \mathrm{v}$, which coincides with a competitive payoff distribution $\nu_{p^{*}}$. The price vector $p^{*}$ in $P$ is given by

$$
p^{*}=\int_{\mathbf{R}^{l}} p(z) d N(z)
$$

where

(5.5) $p$ (z) maximizes $p \cdot z$ over all $p \in P$, for all $z$ in $\mathbf{R}^{l}$, and $N$ is the normal probability measure on $\mathbf{R}^{l}$ with the same first and second moments as $z$, namely, with expectation vector $0=\int_{I} z d \mu$, and covariance matrix

$$
\Sigma=\left(\int_{I} z^{j} \cdot{\underset{\sim}{z}}^{k} d \mu\right)_{j, k=1}^{l}
$$

Proof: Hart [1978].

For a more detailed discussion of this theorem and its conditions, the reader is referred to Hart [1978, Section 3]; it also includes a set of assumptions on $\underset{a}{a}$ and $\left\{u_{t}\right\}_{t \in I}$ implying (5.3).

The theorem also raises the following interesting question: what is the equilibrium price $p^{*}$ that corresponds to the $\mu$-value ( $p^{*}$ is called: value price)?

The first observation is that in this model, the set $P$ of all competitive prices is determined by macro-economic considerations only. Indeed, one needs to know only the aggregated utility function ${ }^{11}$ ) $u_{I}$ and the aggregated (initial) supply $\int_{I} a$, the competitive prices being then exactly the set of super-gradients (i.e., supporting hyperplanes) of $u_{I}$ at $\int_{I} a$ [see Hart, 1978, Corollary 6.19]. All this data is not only "agent

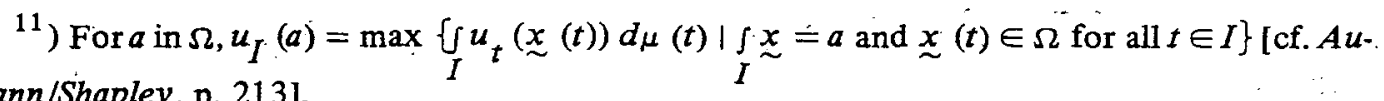
mann/Shapley, p. 213].
} 
free", but also "distribution free" - i.e., the utility function and the income (initial endowment) of any one trader are unspecified; furthermore, not even the distribution of those characteristics in the population need be given. In the case the competitive price is uniquely determined, no problem arises. But which price should be chosen when $P$ is a "large" set? The value considerations point out one such price $p^{*}$ as the customary interpretation of this concept indicates, on grounds of "fairness" and "equity". Obviously, additional data - at the microeconomic level - will be needed for this purpose.

As in the statement of the theorem, let $P$ be the set of all equilibrium prices, $x$ a (fixed) competitive allocation, and $\underset{\sim}{z}=\underset{\sim}{x}-\underset{\sim}{a}$.

Let $S$ be a large random sample (coalition), drawn from the set of traders $I$. If the total supply of $S, \int_{S} \underset{\sim}{a}$, equals its total demand $\int_{S} \underset{\sim}{x}$, then every price vector $p$ in $P$ is also an "S-price", namely an equilibrium price for the economy formed by $S$. In general, however, $\int_{S}(\underset{\sim}{x}-\underset{\sim}{a})=\int_{S} \underset{\sim}{z}$ will be small (by the Law of Large Numbers, since $\int_{I} \underset{\sim}{z}=0$ ), but will not vanish. Therefore, the $S$-prices will be close to $P$; in fact, they will be close to those $p$ in $P \operatorname{maximizing} p \cdot \int_{S} \underset{\sim}{z}$, i.e., following our notation (5.5), to $p\left(\int_{S} z\right)$. Mathematically, this is an easy consequence of the properties of super-gradients [cf. Rockafellar, Theorem 24.6]. Economically, it corresponds to a high price for commodities with a high excess demand, and a low price for those with a low excess demand. It can be also thought of as some kind of an auctioneer's rule in a tatonnement process [cf. Arrow/Hahn, Chapter 11].

Let $Z$ be the distribution of the excess demand $\underset{\sim}{z}$ in the population (i.e., $Z$ is the probability measure on $\mathbf{R}^{l}$ defined by $Z=\mu \circ \widetilde{z}^{-1}$ ). Then, if we choose traders at random, the distribution of their excess demand will be $Z$. However, if we choose samples (coalitions) at random, their aggregated excess demand will no longer be $Z$ distributed. By the Central Limit Theorem, we will get instead (with the adequate normalization) the normal distribution with the same first and second moments as those of $Z$ - namely, $N$.

Combining these two results, and noting that the normalization does not matter, since $p(z)=p(\alpha z)$ for all $\alpha>0$, we finally get: $p^{*}$, as defined by (5.4), is the expected equilibrium price vector of the economy formed by a random subset (or, random sample) of the agents.

A close look reveals that this interpretation actually follows from the value considerations. Indeed, let $d t$ be a small trader, then his value, $(\phi v)(d t)$, is the expected incremental worth ("contribution") of $d t$ to a large sample (coalition), picked at random from the population. Let $S$ be such a sample, then the contribution of $d t$ equals the utility of his allocation, minus its net cost, namely

$$
\left[u_{t}\left({\underset{\sim}{x}}^{S}(t)\right)-p^{S} \cdot\left({\underset{\sim}{x}}^{S}(t)-\underset{\sim}{a}(t)\right)\right] \mu(d t),
$$

where $\left(p^{S}, x^{S}\right)$ is a competitive equilibrium in the economy formed by $S$. By the Law of Large Numbers, $S$ is a "good representation" of the traders space $I$, hence $\underset{\sim}{x^{S}}$. will 
be close to $\underset{\sim}{x}$ (our fixed competitive allocation for the whole economy ${ }^{12}$ ). Taking expectation in (5.7) we get (in the limit)

$$
(\phi \mathrm{v})(d t)=\left[u_{t}(\underset{\sim}{x}(t))-E\left(p^{S}\right) \cdot \underset{\sim}{z}(t)\right] \mu(d t),
$$

therefore the value payoff distribution is competitive, and the corresponding price $p^{*}$ is precisely $E\left(p^{S}\right)$, i.e., the expected equilibrium price for a random sample (coalition).

\section{References}

Arrow, K.J., and F.H. Hahn: General Competitive Analysis, San Francisco 1971.

Aumann, R.J.: Markets with a Continuum of Traders. Econometrica 32, 1964, 39-50.

-: Values of Markets with a Continuum of Traders. Econometrica 43, 1975, 611-646.

Aumann, R.J., and M. Kurz: Power and Taxes in a Multi-Commodity Economy. Israel Journal of Mathematics 27, 1977, 185-234.

Aumann, R.J., and M. Perles: A Variational Problem Arising in Economics. Journal of Mathematical Analysis and Applications 11, 1965, 488-503.

Aumann, R.J., and L.S. Shapley: Values of Non-Atomic Games. Princeton 1974.

Champsaur, P.: Cooperation Versus Competition. Journal of Economic Theory 11, 1975, 394-417.

Debreu, G., and H. Scarf: A Limit Theorem on the Core of an Economy. International Economic Review 4, 1963, 235-246.

Harsanyi, J.: A Bargaining Model for the Cooperative $n$-Person Game. Annals of Mathematical Studies 40, 1959, 325-355.

Hart, S.: Asymptotic Value of Games with a Continuum of Players. Journal of Mathematical Economics 4, 1977a, 57-80.

-: Values of Non-Differentiable Markets with a Continuum of Traders. Journal of Mathematical Economics 4, 1977b, 103-116.

-: Measure-Based Values of Market Games. TR\# 254, Economics Series. Institute for Mathematical Studies in the Social Sciences, Stanford University, 1978; forthcoming in Mathematics of Operations Research.

Hildenbrand, W.: Core and Equilibria in a Large Economy. Princeton 1974.

Kannai, Y.: Values of Games with a Continuum of Players. Israel Journal of Mathematics 4, 1966, $54-58$.

Mas-Colell, A.: Competitive and Value Allocations of Large Exchange Economies. Journal of Economic Theory 14, 1977, 419-438.

Rockafellar, R.T.: Convex Analysis. Princeton 1970.

Shapley, L.S.: Values of Large Games VII: A General Exchange Economy with Money. RM-4248-PR, The Rand Corporation, Santa Monica, California, 1964.

-: Utility Comparison and the Theory of Games. La Décision. Paris 1969.

Vind, $K$.: Edgeworth Allocations in an Exchange Economy with Many Traders. International Economic Review 5, 1964, 165-177.

\footnotetext{
$\left.{ }^{12}\right)$ The non-uniqueness of $\underset{\sim}{x}$ is "inessential": by $(2.7), u_{t}(\underset{\sim}{x}(t))-p \cdot(\underset{\sim}{x}(t)-\underset{\sim}{g}(t))$ does not depend on the particular t.u.c.e. allocation $\underset{\sim}{x}$ chosen. Therefore, only the non-uniqueness of the equilibrium price vectors matters in getting (5.8) from (5.7).
} 